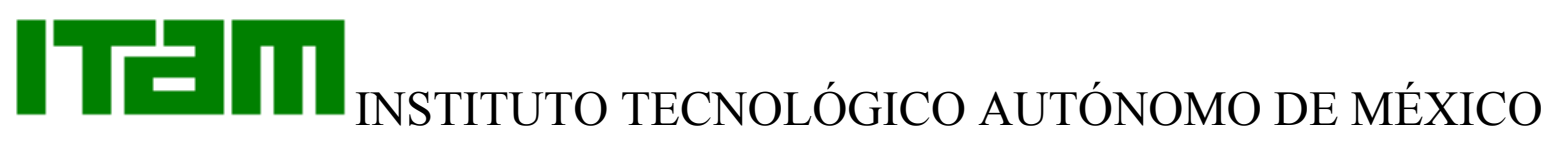

\section{CENTRO DE INVESTIGACIÓN ECONÓMICA}

\section{Discussion Paper Series}

Corruption, Extortion, and the Boundaries of the Law

Svetlana Andrianova

University of Leicester

and

Nicolas Melissas

Instituto Tecnológico Autónomo de México

September 2006

Discussion Paper 06-05

Av. Camino a Santa Teresa \# 930

Col. Héroes de Padierna

México, D.F. 10700

$\mathrm{M}$ E X I C O 


\title{
Corruption, Extortion, and the Boundaries of the Law*
}

\author{
Svetlana Andrianova ${ }^{\dagger} \quad$ Nicolas Melissas ${ }^{\ddagger}$
}

September 8, 2006

\begin{abstract}
We consider a set-up in which a principal must decide whether or not to legalise a socially undesirable activity. The law is enforced by a monitor who may be bribed to conceal evidence of the offence and who may also engage in extortionary practices. The principal only declares the activity illegal if the activity is "very harmful" and if the private benefit (received by the agent if she breaks the law) is "high". We present comparative static results and highlight policy implications.
\end{abstract}

JEL-codes: D82, L22, K4.

Keywords: Moral Hazard, Collusion, Non-contractible Output, Rewards and Punishments.

${ }^{*}$ We would like to thank without implicating Cecile Aubert, John Conley, Gianni De Fraja, Panicos Demetriades, Mathias Dewatripont, Paul Heidhues, Maurice Kugler, Claudio Mezzetti and Tim Worrall, as well as seminar participants at the University of Leicester, for helpful comments and discussions. Earlier versions of the paper were presented at the European meeting of the Econometric Society in Berlin, Royal Economic Society annual conference in Nottingham, Econometric Society Far Eastern Meeting in Singapore and International Economic Association congress in Buenos Aires.

${ }^{\dagger}$ University of Leicester, University Road, Leicester LE1 7RH, UK. E-mail s.andrianova@le.ac.uk.

${ }^{\ddagger}$ Corresponding author. Centro de Investigación Económica, Instituto Tecnológico Autónomo de México, Camino a Santa Teresa 930, México D.F. 10700, Mexico. E-mail nmelissas@itam.mx. Tel + 52.55.56.28.40.00 Fax + 52.55.56.28.40.58 


\section{Introduction}

Corruption is - and has always been - a source of concern for policy makers. For example, Theodore Roosevelt in a 1903 speech said: "we must show our abhorrence of ... corruption, in public and in private life". ${ }^{1}$ More recently, in 1999 the World Bank president James Wolfensohn asserted that: “... so far as [the World Bank] is concerned, there is nothing more important than the issue of corruption". ${ }^{2}$ Similar words also came out of the mouth of George Bush in the wake of recent scandals that shook corporate America. Policy makers have good reasons to put the fight against corruption high on the political agenda: Mauro (1995) showed that corrupt countries experience lower growth and James Wolfensohn argued that it is the poor who suffer the most from it.

Obviously, corruption can take many forms as it is prevalent in the political, corporate and criminal spheres of society. To illustrate the forms of corruption that we will study in this paper, consider the following examples. ${ }^{3}$ In the 1960s and early 1970s the Hong Kong police was very corrupt and regularly received bribes in order not to report illegal gambling activities. Prior to 1975 the Philippines' Bureau of Internal Revenue was renowned for its corrupt tax inspectors. In one famous example two tax inspectors found evidence of a 2 million pesos tax fraud. Instead of reporting this to their superiors, they went to the contravening company and asked for one million pesos in exchange for their silence. In the early 1970s Singapore Custom officials were given bribes in order to allow some illegal imports. What those examples share in common is that people receive bribes in order to conceal evidence of illegal behaviour. Henceforth we refer to this practice as ex post corruption. Ex post refers to the fact that the collusive agreement arose after the occurrence of the illegal activity.

\footnotetext{
${ }^{1}$ Address at the Dedication Ceremonies of the Louisiana Purchase Exposition on April 30, 1903. http://www.jmu.edu/madison/center/main_pages/madison_archives/life/secretary/la_purchase/roosevelt.htm

${ }^{2}$ Plenary Address at the 9th International Anti-Corruption Conference in Durban, South Africa, 9-15 October, 1999. http://ww1.transparency.org/iacc/9th_iacc/papers/day1/plenary/d1pl_jwolfensohn.html

${ }^{3}$ All examples in this paragraph are taken from Klitgaard (1988).
} 
While ex post corruption has been extensively studied in the economics literature (see below for more details) other types of corruption have received scant attention. For example, in Hong Kong the police force had to give the impression that they were serious about tackling illegal gambling. To save appearances they colluded with the gambling mafia to produce "fake" evidence of their efforts to curb illegal gambling activities as explained in the following quote:

An arrangement [between the Hong Kong police and the gambling mafia] would sometimes be made for paid "actors" to substitute for the casino's staff and regular customers. When arrested, the actors had their fines paid by the [mafia] and were released after a night in jail. (Klitgaard, 1988, p. 99)

Henceforth we refer to this practice as ex ante corruption. In contrast with ex post corruption, the collusive agreement here arose before the occurrence of the illegal activity.

In a related vein, examples abound in which one party creates fake evidence to indict another. For example, in Lahore (Pakistan) police officers are notorious for putting drugs in the hotel rooms of unsuspecting tourists. The tourists then have to bribe the police officers to avoid going to jail. Similarly, the corrupt Philippino tax inspectors did not solely receive bribes to hide evidence of the offence. Instead, they also pretended that some taxpayers had understated their taxable income even if this was not true. Some gullible taxpayers believed that they had made an error in their income statement and then bribed the tax official to induce him not to report this "error" to his superiors. The Singapore Custom officials also engaged in similar activities. Practices of this kind illustrate what we model as extortion in this paper. Note that extortion is crucially different from ex ante corruption: in the case of the latter, the Hong Kong police force needs the mafia's consent (and the participation of actors) to create evidence of illegal behaviour. If the police officer extorts, however, then he creates (fake) evidence of the offence without the tourist's consent. Observe also that we define extortion as planting (fake) evidence of an offence regardless of whether the tourist pays a bribe or ends up in jail. 
Suppose you are the president of Pakistan. How should you deter extortion and ex post corruption? Should you raise penalties? Should you raise rewards for reporting evidence of illegal behaviour? Should you instead go for a more drastic solution and legalise the use of drugs? ${ }^{4}$ Alternatively, suppose you are the Hong Kong governor. How should you deter ex ante and ex post corruption? In this paper we develop a principal-monitor-agent model to tackle these questions. More specifically, we consider a set-up in which an agent chooses whether or not to undertake a socially undesirable activity. ${ }^{5}$ The agent derives a private benefit from undertaking the activity. The principal (i.e. the social planner) must decide (i) whether or not to declare the activity illegal and if so (ii) the jail sentence to be imposed on the agent and the reward to be given to the monitor in case the monitor reports evidence of the offence. The monitor can either uncover evidence of illegal behaviour by undertaking costly monitoring or he can plant fake evidence of the offence. First, (in Section 3) we assume that the monitor does not need the agent's consent to plant fake evidence (as is the case with extortion). Next (in Section 4) we assume that the monitor needs the agent's consent (or her active participation) to create the evidence of the offence.

Our analysis of ex post corruption and extortion suggests that the principal only wants to declare an activity illegal if the activity is "very harmful" and if the private benefit is "high". To understand this result, suppose the private benefit is "not high" and that the principal declares the activity illegal. In our model if the monitor catches an offending agent, he can prevent the agent from consuming her private benefit. ${ }^{6}$ An of-

\footnotetext{
${ }^{4}$ The U.S. interwar experience nicely illustrates that governments sometimes prefer to legalise undesirable activities. As is well known, alcohol prohibition led to widespread corruption and little law abiding behaviour. This induced some states to legalise the consumption of alcohol in the 1930's.

${ }^{5}$ For example, the activity could be: smoking cannabis, drinking alcohol, gambling, setting up an illegal casino, etc.

${ }^{6}$ For example, if a police officer catches a tourist in the possession of cannabis, he will give the drugs to his supervisor as "proof" that the tourist broke the law. In that case the tourist will never smoke her cannabis (and will thus never get her private benefit). Our results do not crucially hinge on our assumption that $M$ can prevent $A$ from consuming her private benefit with probability one. If the
} 
fending agent has therefore two good reasons to bribe the monitor. First, she wants to avoid a jail sentence and second, she wants to consume her private benefit. If the agent is framed, however, she only wants to bribe the monitor to avoid going to jail. Hence, in equilibrium the monitor will receive a bigger bribe if he catches an offending agent than if he decides to frame her. The greater the private benefit, the greater the difference between the two bribes. Furthermore, we assume that monitoring is a costly activity while extorting is not. Hence, if the private benefit is "not high", the bigger bribe does not compensate the monitor for his disutility of effort and he never monitors. The agent knows this and therefore always breaks the law. As the difference between the two bribes does not depend on the harmfulness of the illegal activity, this result holds even if the illegal activity were infinitely harmful. Suppose now that the private benefit is "high" and that the activity is declared illegal. Then, the agent and the monitor play a mixed-strategy Nash equilibrium in which the agent randomises between breaking the law and not breaking the law, while the monitor randomises between extorting and providing effort to uncover evidence of the possible offence. The principal knows this. She also knows that by changing the punishment-reward structure she influences both equilibrium probabilities. If the monitor exerts effort, welfare decreases by the disutility of monitoring. However, monitoring also induces the agent to abide by the law, which raises welfare. If the activity is not "very harmful", the first effect dominates the second one. In that case the principal wants to set the punishment-reward structure such as to implement an equilibrium in which the monitor never exerts effort. We show that this cannot be implemented by any finite punishment-reward structure. Hence, the principal strictly gains by declaring the activity legal. However, if the activity is "very harmful", then the second effect dominates the first one and the principal (strictly) gains by declaring the activity illegal.

monitor could only prevent the agent from consuming her private benefit with probability $p>0$, then the expected bribe he gets if he catches an offending agent would still be bigger than the bribe he gets if he were to frame an innocent individual, and all our results should go through. A more detailed discussion of this assumption is provided in section 3 . 
We also show that the agent is more likely to abide by the law if the monitor has a lot of bargaining power, or if the disutility of monitoring is small, or if the agent derives a big private benefit from the illegal activity. Finally, we show that if the monitor needs the agent's consent to create the evidence of illegal behaviour (i.e. in the case of ex ante corruption), then the principal can always implement an outcome close to first best by punishing the agent infinitely hard.

Our paper belongs to the literature on collusion (or corruption) when the principal cannot contract on output. ${ }^{7}$ No paper in that literature has found that the principal can gain by legalising a socially undesirable activity. Moreover, only Hindriks et al. (1999) and Polinsky and Shavell (2001) allow the monitor to extort and to engage in ex post corruption. ${ }^{8}$ In Polinsky and Shavell (2001) it is never optimal to legalise the undesirable activity because it is implicitly assumed that both corruption and extortion activities are costless. In contrast, we find that the principal prefers to legalise the activity precisely because monitoring is more costly (in terms of effort) than extorting. Hindriks et al. (1999) analyse a set-up in which a tax inspector can extort and engage in ex post corruption. However, they are primarily interested in studying the efficiency and equity consequences of different tax schemes which is not the purpose of this paper.

Another strand in the literature on collusion (or corruption) in three-tier organizations considers a set-up with adverse selection and moral hazard in which the principal can

\footnotetext{
${ }^{7}$ See, among others, Strausz (1997), Hindriks et al. (1999), Carrillo (2000a, 2000b), Polinsky and Shavell (2001), Mishra (2002).

${ }^{8}$ Strausz (1997) analyses a similar set-up as ours except that he does not allow the monitor to extort or engage in ex ante corruption. In Carrillo (2000a, 2000b) the monitor's wage is independent of his report and he also restricts attention to the ex post corruption case. Mishra (2002) considers a two-monitor set-up and, using efficiency as a benchmark, compares a vertical hierarchy (i.e. the second monitor monitors the first one) with a horizontal one (i.e. both monitors monitor the agent). As in the previous papers, monitors do not extort nor engage in ex-ante corruption.
} 
contract on the agent's output. ${ }^{9}$ In particular, Kofman and Lawarrée (1993) have shown that the principal may prefer not to hire the monitor ${ }^{10}$ if her signal is sufficiently imprecise or if the agent's maximal punishment is low. We show that their negative result also holds if the principal cannot contract on output provided that the monitor can extort and engage in ex post corruption. Moreover, in our model the principal may prefer to legalise the activity even if the monitoring technology is perfect and if the agent's maximal punishment is arbitrarily high. Khalil and Lawarrée (2003) assume that the principal cannot commit ex ante to hiring the monitor after the output realisation. They show that, due to conflicting non-commitment and ex post corruption constraints, the principal may also prefer to legalise the undesirable activity. In contrast, our result does not rely on a commitment problem.

\section{The benchmark model without corruption or extortion}

An agent $(A)$ must choose whether or not to undertake an activity. If she does not undertake the activity, this results in welfare $\bar{w}$ and a zero payoff to $A$. If she undertakes the activity, this leads to welfare $\underline{w}$ and, if undetected, to a private benefit of $\pi>0 .{ }^{11}$ The activity is socially undesirable in the sense that $\underline{w}+\pi<\bar{w}$. The principal $(P)$ must decide whether or not to legalise the activity. If she declares the activity illegal, she must rely on a monitor $(M)$ to enforce the law. $M$ 's outside option is equal to zero and he is protected by limited liability. If $M$ decides to monitor, he suffers a disutility normalised to 1 . If $M$ monitors when $A$ broke the law, then he finds evidence of $A$ 's offence with probability one. ${ }^{12}$ If $M$ decides not to monitor, his disutility is zero and $A$ 's possible

\footnotetext{
${ }^{9}$ See, among others, Kofman and Lawarrée (1993), Kessler (2000), Khalil and Lawarrée (2003), as well as the classic paper by Tirole (1986).

${ }^{10}$ In our model this corresponds to legalising the undesirable activity.

${ }^{11} \pi$ is expressed in monetary terms. $\pi$ can also be interpreted as a sum of money generated by $A$ 's activity. We will come back to this interpretation on page 20.

${ }^{12}$ It may be more realistic to assume that $M$ only finds evidence of the offence (when $A$ broke the law) with probability $\lambda<1$. Setting $\lambda=1$, however, simplifies the exposition without affecting our results.
} 
offence remains undetected. $M$ 's choice of action is not contractible. If $M$ does not present any evidence of the offence to $P$, then w.l.o.g. we assume that $P$ compensates $M$ with a zero transfer. ${ }^{13}$ Otherwise, if $M$ presents evidence of the offence, then $P$ transfers reward $r$ to $M$ and puts $A$ for $x$ years in jail. ${ }^{14}$ Let $f$ be the monetary equivalent of the jail sentence (i.e. $A$ is indifferent between paying a fine $f$ and spending $x$ years in jail). As a convention, in what follows we shall assume that $P$ and $A$ are female, while $M$ is male.

Let $\alpha \in[0,1]$ and $\mu \in[0,1]$ denote the probability with which $A$ abides by the law and $M$ monitors, respectively. $P$ 's objective is to maximise social welfare by optimally choosing whether or not to legalise the activity. In the analysis of this problem, we adopt the following tie-breaking rule: if indifferent between legalising and not legalising the activity, $P$ will legalise it. ${ }^{15}$ If $P$ declares the activity legal, then, straightforwardly, social welfare is $\underline{w}+\pi$. If, however, $P$ declares the activity illegal, then she faces the following problem:

$$
\begin{gathered}
\max _{r, f} \underline{w}+\alpha^{*} \Delta w-\mu^{*}+\left(1-\alpha^{*}\right)\left[\left(1-\mu^{*}\right) \pi-\mu^{*}(r+f)\right] \\
\text { s.t. } \\
\alpha^{*} \in \arg \max \quad(1-\alpha)\left[\left(1-\mu^{*}\right) \mu^{*} r-\mu^{*} \geq 0\right. \\
\mu^{*} \in \arg \max \quad\left(1-\mu^{*}\right) \mu r-\mu \\
f, r \geq 0 \\
\alpha, \mu \in[0,1]
\end{gathered}
$$

\footnotetext{
${ }^{13}$ This is w.l.o.g. because rewarding $M$ for reporting no evidence only decreases his incentives to monitor. Hence, in the absence of any evidence, $P$ should give the lowest possible reward to $M$. As $M$ is protected by limited liability and possesses a zero outside option, his lowest possible reward is equal to zero.

${ }^{14}$ We assume that $P$ can only punish $A$ by sending her to jail. We will argue below (see Section $3, \mathrm{p}$. 20) that this is without loss of generality.

${ }^{15}$ This rule is reasonable if making the activity legal frees up the resources for other enforcement activities without affecting the equilibrium behaviour. This is, indeed the case in the results we find below (see also further discussion on p. 16).
} 
(3) and (6) ensure that $A$, taking $\mu^{*}$ and $f$ as given, chooses $\alpha$ to maximise her utility. Similarly, (4) and (6) ensure that $M$, taking $r$ and $\alpha^{*}$ as given, chooses $\mu$ optimally. Equation (1) states that $P$, correctly anticipating how $r$ and $f$ affect $\alpha^{*}$ and $\mu^{*}$, chooses them optimally subject to $M$ 's individual rationality constraint, (2), and the limited liability constraint, (5). Notice that the expected welfare is decreasing in $r$, which is justified by the observation that in order to pay $r, P$ has to raise money, e.g. through taxation, which entails an inefficiency (due to costly collection of taxes, etc), and the inefficiency is increasing with the size of the transfer.

It is clear that the statement of $P$ 's constrained optimisation above takes into account the moral hazard on the part of $M$ and $A$, but rules out the possibility of either extortion or corruption, which is the focus of the next section. Here, however, we note the following benchmark result.

Proposition 1 In a corruption-free and extortion-free world, $P$ declares the activity illegal, $f^{*}$ and $r^{*}$ are arbitrarily large, $1-\alpha^{*}$ and $\mu^{*}$ are close to zero, and the expected welfare is close to $\bar{w}$.

To understand the intuition behind Proposition 1, suppose $P$ declares the activity illegal. If $A$ anticipates that $M$ will exert monitoring effort, she prefers to abide by the law as she dislikes going to jail. If $A$ anticipates $M$ to do nothing, she prefers to break the law as she knows that she will then get $\pi$. Similarly, $M$ does not want to incur a disutility when he anticipates that $A$ abides by the law. However, monitoring becomes very profitable if $A$ always breaks the law. Hence, $A$ and $M$ play a mixed-strategy Nash equilibrium. $P$ knows this. In equilibrium $\alpha^{*}$ is increasing in $r$, which is intuitive: the higher $r$, the higher $M$ 's incentives to monitor; to make $M$ indifferent, $A$ must reduce her probability of breaking the law. Similarly, $\mu^{*}$ is decreasing in $f$, which is also intuitive: the higher $f$, the lower $A$ 's incentives to break the law; to make $A$ indifferent, $M$ must reduce his probability of monitoring. Hence, the social planner can come close to first-best by setting $r$ and $f$ at an arbitrarily high level. 


\section{The model with ex post corruption and extortion}

We now allow $M$ to extort and to engage in ex post corruption. Extortion is modeled as the alternative to monitoring: $M$ can choose to either monitor with probability $\mu \in[0,1]$ or to extort with probability $1-\mu \cdot{ }^{16}$ To motivate this choice, consider our example of extortion in Lahore (see Introduction). If the police officer decides to extort, he must first wait until the tourist is out of her hotel, plant the evidence in the hotel room, wait for the tourist to return, bargain with the tourist over a possible bribe, bargain with the hotel room owner over how the tourist's bribe should be divided, etc. All these activities take time. The police officer could have used that time to find genuine proof of the tourist breaking the law.

In what follows, we crucially assume that monitoring is more costly (in terms of effort) than extorting. We defend this assumption on the grounds that finding proof of misconduct can be a lengthy, difficult and even dangerous job. For example, it may involve laboratory tests (such as investigating finger prints, blood samples, etc), interrogations, tapping phone conversations, shadowing people, and such like. Obviously, extortionary activities also require effort, but one would not expect them to be as costly as the activities required to uncover proof of misconduct. Let $d_{\text {monitor }}$ and $d_{\text {extort }}$ denote the disutility of, respectively, monitoring and extorting. In this paper, we assume that $d_{\text {monitor }}=1$ and $d_{\text {extort }}=0$. However, we expect our main result to hold as long as $d_{\text {monitor }}>d_{\text {extort }}$.

If $M$ chooses to extort, then $A$ gets framed with probability one. ${ }^{17}$ We motivate our choice of payoffs in this case on the basis of our leading example. Suppose the tourist leaves her hotel room at 1 p.m. At 1.05 p.m. the police officer puts the drugs in the tourist's hotel room and waits for the tourist to return. Suppose the tourist returns at 4

\footnotetext{
${ }^{16}$ In fact, in addition to the choice between monitoring and extorting, we could allow $M$ to do nothing. However, this extra option will never be chosen by $M$, as it is clearly dominated by the option to extort.

${ }^{17}$ Allowing $A$ to be framed with a probability less than one does not qualitatively change the results.
} 
p.m. and that, between 1 p.m. and 4 p.m., she did not break the law. The tourist and the police officer then bargain over a possible bribe. If the tourist successfully bribes the police officer, they get (at 4.05 p.m.) -bribe and bribe, respectively. If the tourist does not succeed to bribe the police officer, they get $-f$ and $r$, respectively. Suppose now that the tourist bought cannabis at 2 p.m. We then assume that the tourist smokes her cannabis immediately. ${ }^{18}$ Hence, at 4 p.m. $\pi$ has already been consumed and does not affect the size of the bribe. Thus at 4.05 p.m., it is either the case that the tourist successfully bribed the police officer, which gives $\pi$ - bribe to the former and bribe to the latter, or it is the case that the tourist ends up in jail, which leaves the tourist with $\pi-f$ and the police officer with $r$.

If $M$ chooses to monitor, then detection of $A$ 's offence (if committed) is certain, as before. If $A$ abides by the law, she gets zero while $M$ gets -1 (i.e. the disutility of monitoring). ${ }^{19}$ More interestingly, suppose $M$ monitors while $A$ breaks the law. In that case we assume that $M$ can physically prevent $A$ from getting $\pi$. This assumption is natural: suppose the tourist leaves her hotel at 1 p.m. She does not realise that she is being followed by a diligent police officer and, at 2 p.m., buys cannabis. Then it will be optimal for the police officer to "catch" the tourist before she consumes her drugs. For in that case the police officer can say: "If I report this offence to $P$, you get $-f$ and you cannot enjoy your cannabis". Hence, the police officer gets a bigger bribe if he catches the tourist committing the offence as compared to the bribe he gets if he extorts the tourist. Let $\beta \in[0,1]$ denote the bargaining power of $A$, and $b$ denote the bribe $A$ pays to $M$. We now know enough to state and prove the following Lemma.

\footnotetext{
${ }^{18}$ At 2 p.m. the tourist knows that she was not caught red-handed by the police officer. Hence, she knows that the police officer will be waiting for her in her hotel room at 4 p.m. If there is a small probability that she will be subject to a body search, she will prefer to smoke her cannabis immediately.

${ }^{19}$ We do not allow the police officer to frame the tourist if he realises at 4 p.m. that she did not break the law during the day. In our model the monitor can only frame the agent after a certain mise en scène, such as planting the drugs in her hotel room during the day. This assumption realistically describes the way extortion occurs in reality, including our earlier example of Lahore.
} 
Lemma 1 Suppose $A$ is caught while she broke the law. She will then successfully bribe $M$ if and only if $\pi+f-r \geq 0$, and the bribe will be equal to $r+(1-\beta)(\pi+f-r)$. Suppose $A$ is framed. She will then successfully bribe $M$ if and only if $f-r \geq 0$, and the bribe will be equal to $r+(1-\beta)(f-r)$.

Proof: Suppose $A$ broke the law while $M$ monitored. Note that if no evidence is reported when $A$ offended and $M$ monitored, then $A$ gets $\pi-b$ while $M$ gets $b$, making the joint surplus of $\pi$. Otherwise, if the evidence is reported when $A$ offended and $M$ monitored, then $A$ gets $-f$ while $M$ gets $r$, which gives the joint surplus of $r-f$. Ex post corruption will take place whenever there is a non-negative excess surplus from concealing the evidence as opposed to declaring it ${ }^{20}$, namely $\pi+f-r \geq 0$, with $A$ paying $1-\beta$ of this surplus to $M$ (on top of $r$ ) as the bribe.

Following similar reasoning in the case of extortion, there are also two possibilities. If $f-r \geq 0, A$ will pay a bribe and will not go to jail. If $f-r<0, A$ will end up in jail after being framed. To check, note that $A$ who offended and is being framed will by assumption continue to enjoy the benefit of her offence, $\pi$, regardless of whether she ends up in jail or not. So, if $A$ is being framed when she offended, then extortionary payment prevents the report of the evidence and gives $\pi-b$ to $A$ and $b$ to $M$. If there is no extortionary payment, then $M$ reports the fake evidence to $P$ and $A$ gets $\pi-f$ while $M$ gets $r$. Thus, a framed agent will engage in the extortionary agreement if there is a positive excess surplus from declaring no evidence in place of the fake evidence, namely $\pi-(\pi-f+r) \geq 0 \Leftrightarrow f-r \geq 0$. If $A$ is being framed when she did not offend, then following the report of no evidence, $A$ and $M$ get $-b$ and $+b$, respectively, while following the report of the fake evidence $A$ and $M$ get $-f$ and $r$, resp. Hence, the extortionary agreement will take place if and only if $f-r \geq 0$, with $A$ paying $1-\beta$ of this surplus to $M($ on top of $r$ ) as the bribe.

\footnotetext{
${ }^{20}$ We assume w.l.o.g. that if $M$ is indifferent between accepting and not accepting the bribe, he accepts the bribe (and $A$ does not go to jail).
} 
Lemma 1 contains two important insights. First, it proves that if $M$ catches an offending agent, he gets a bigger bribe than the one he gets if he frames an innocent individual. Second, it proves that the difference between the two bribes is increasing in $\pi$. As those insights are crucial in the proofs of our next propositions, it is important to realise that they do not rely on our assumption that if $A$ is caught breaking the law, $M$ can prevent her from consuming $\pi$ with probability one. To see this, denote $b_{\text {monitor }}$ and $b_{\text {extort }}$ as the the bribes $M$ gets if he, respectively, catches an offending agent and frames an innocent individual. Moreover, suppose that if $A$ breaks the law (while $M$ monitors), then with probability $p>0 M$ catches her before she can consume $\pi$ (i.e. when she still possesses the cannabis), while with probability $1-p \quad M$ catches her after she consumed $\pi$ (i.e. after she smoked the cannabis). In that case, $M$ 's expected bribe (conditional on $A$ breaking the law) equals

$$
p[r+(1-\beta)(\pi+f-r)]+(1-p)[r+(1-\beta)(f-r)],
$$

and $b_{\text {monitor }}-b_{\text {extort }}$, conditional on $A$ breaking the law, remains positive and increasing in $\pi$. At the end of this section (see p. 20), we argue, additionally, that both insights should remain valid under the interpretation that $\pi$ represents a sum of money and that $M$ has no information about $A$ 's level of wealth.

Define the corruption and framing surplus, respectively, as:

$$
C S=\max \{0, \pi+f-r\} \quad \text { and } \quad F S=\max \{0, f-r\}
$$

If $P$ declares the activity illegal, her optimisation problem with corrupt and possibly extorting $M$ becomes:

$$
\begin{array}{cc}
\max _{r, f} & \underline{w}+\alpha^{*} \Delta w-\mu^{*}+\left(1-\alpha^{*}\right)\left[\left(1-\mu^{*}\right)+\mu^{*} \cdot I_{\{\pi+f-r \geq 0\}}\right] \pi- \\
& -(f+r)\left[\left(1-\alpha^{*}\right) \mu^{*} \cdot I_{\{\pi+f-r<0\}}+\left(1-\mu^{*}\right) \cdot I_{\{f<r\}}\right] \\
\text { s.t. } & \mu^{*}\left(\left(1-\alpha^{*}\right)[r+(1-\beta) \cdot C S]-1\right)+\left(1-\mu^{*}\right)[r+(1-\beta) \cdot F S] \geq 0 \\
& \alpha^{*} \in \arg \max (1-\alpha)\left[\left(1-\mu^{*}\right) \pi+\mu^{*} \beta \cdot C S\right]-\left(1-\alpha \mu^{*}\right) f+\left(1-\mu^{*}\right) \beta \cdot F S(10)
\end{array}
$$




$$
\begin{gathered}
\mu^{*} \in \arg \max \mu\left(\left(1-\alpha^{*}\right)[r+(1-\beta) \cdot C S]-1\right)+(1-\mu)[r+(1-\beta) \cdot F S](11) \\
f, r \geq 0 \\
\alpha^{*}, \mu^{*} \in(0,1)
\end{gathered}
$$

where the indicator symbol $I$ equals 1 if its subscripted condition is satisfied and 0 otherwise. In this programme, the prospect of ex post corruption and extortion are accounted for by the inclusion of $C S$ and $F S$ given by (7). Compared to the benchmark case, the objective function in (8) now differs in the last two terms with square brackets. The first set of square brackets in (8) reflects the probability with which offending $A$ expects to enjoy the private benefit of her offence, $\pi$ : if either $M$ chooses to extort, or if $M$ chooses to monitor and subsequently agrees to hide the evidence. The second set of square brackets in (8) reflects the only two cases when $M$ gives the evidence to $P$ and hence when the expected welfare declines by $f+r$ : either A cannot gain by bribing $M$ when $M$ chose to monitor and found the evidence of $A$ 's offence (i.e. $\pi+f-r<0$ ), or A cannot gain by bribing $M$ when $M$ chose to extort (i.e. $f<r$ ). Constraints (10), (11) and (13) account for the simultaneous move game played between $A$ and $M$. That is, (10) and (13) ensure that $A$, taking $\mu^{*}$ and the possibility of corruption and extortion as given, chooses $\alpha$ to maximise her utility. Similarly, (11) and (13) ensure that $M$, taking $\alpha^{*}$ and the possibility of corruption and extortion as given, chooses $\mu$ optimally to maximise his expected utility. The participation constraint of $M$ is (9) and it states that $M$ 's expected gain, given his choice of $\mu^{*}$ and $A$ 's choice of $\alpha^{*}$, should not be negative. Note that the programme does not include any constraint on $A$ 's participation: we implicitly assume that $A$ cannot help but be involved in this contract either as a citizen of the country in which corruption and extortion take place, or as a tourist who willingly accepts a small chance of extortion because of the significant enjoyment she gets from sightseeing in the exotic destination. ${ }^{21}$

Proposition 2 If $\pi<\frac{1}{1-\beta}, P$ legalises the activity.

\footnotetext{
${ }^{21}$ We study the case of explicit constraint on $A$ 's participation in Section 4.
} 
Proof: Suppose $P$ declares the activity illegal. Observe from (11) that $M$ 's gain from monitoring is decreasing in $\alpha: \partial^{2} E U_{M} / \partial \mu \partial \alpha<0$ where $E U_{M}$ is the expected utility of $M$. This is intuitive: if $A$ is more likely to abide by the law, it becomes less profitable to monitor. Suppose that $M$ 's incentives to monitor are maximal, i.e. that $\alpha=0$. In that case $\mu^{*}=0$ if $C S-F S<\frac{1}{1-\beta}$. The LHS of this last inequality is less than, or equal to, $\pi$. Hence, if $\pi<\frac{1}{1-\beta}, M$ will always extort. As $\mu^{*}=0, \alpha^{*}=0$. It follows from (8) that $P$ 's maximization problem then boils down to

$$
\max _{r, f} \quad \underline{w}+\pi-(f+r) I_{\{f<r\}}
$$

which is maximal whenever $f \geq r$. Note that $P$ is indifferent between legalising and not legalising the activity as in both cases she gets $\underline{w}+\pi$. Our result then follows from our tie-breaking rule.

To understand the intuition behind this result, suppose $P$ declares the activity illegal and that $f \geq r$. Then $F S$ and $C S$ are both non-negative and $A$ would gain if she bribes $M$ when there exists (possibly, fake) evidence of the offence. It transpires from (11) that $M$ has two good reasons to extort: (i) monitoring is costly in terms of effort while extorting is not and (ii) while extortion always secures a non-negative payoff, monitoring is expected to result in a negative payoff some of the time (since if $M$ monitored and $A$ did not break the law, $M$ suffers the monitoring disutility). In contrast, there is one good reason to monitor: if $M$ catches the offending agent, he receives a bigger bribe. As mentioned previously, this is based on our assumption that if $M$ catches an offending agent, he can-by reporting this to $P$-prevent $A$ from enjoying $\pi . A$ knows this and is therefore willing to pay a higher bribe than if she had been framed. The higher $\pi$ the higher the difference between the two bribes and the higher $M$ 's incentives to monitor. If $\pi$ is "low", the difference between the two bribes is not enough to compensate $M$ for his disutility of monitoring and he-independently of $\alpha$-always extorts. $A$ knows this and therefore prefers to always break the law. The fact that $M$ always extorts, however, does not reduce welfare when $f \geq r$ : it merely represents a transfer of wealth between two risk-neutral players. Hence, $P$ is indifferent between legalising and not legalising the 
activity. We believe that in this case it is reasonable to assume that $P$ will declare the activity legal. The reasoning here is this: If $P$ declares the activity illegal, this does not deter $A$ from breaking the law. Instead, this only induces $M$ to devote a rather large fraction of his time to extortionary activities. If $P$ legalises the activity, however, $M$ could devote more time to other law enforcement activities (such as catching thieves, policing the streets, detecting road traffic violations, etc).

$P$ 's problem becomes more interesting as soon as $\pi>\frac{1}{1-\beta}$, as illustrated in the following Remark 1 If $\pi>\frac{1}{1-\beta}, P$ is no longer indifferent between legalising and not legalising the activity.

Proof: When $P$ chooses between legalising and not legalising the activity, she will be indifferent between the two options only if both deliver the same outcome: $A$ always breaks the law, $M$ does not monitor and $A$ never goes to jail. The implication of $A$ never going to jail is that $f \geq r$ and hence that $C S=\pi+f-r$ and $F S=f-r$. By contradiction, suppose $P$ declares the activity illegal, that $(\alpha, \mu)=(0,0)$ constitutes an equilibrium in the simultaneous move game played between $A$ and $M$ and that $A$ never goes to jail. Suppose $M$ expects $\alpha=0$. If he monitors, he gets $r+(1-\beta)(\pi+f-r)-1$. If he extorts, he gets $r+(1-\beta)(f-r)$. It is a best response for $M$ to extort if $\pi \leq \frac{1}{1-\beta}$, which contradicts the assumed inequality.

The intuition here mirrors the intuition provided above for Proposition 2: if $\pi$ is "high", the difference between the two bribes is enough to compensate $M$ for his disutility of monitoring. Hence, if $P$ declares the activity illegal and if $M$ anticipates $A$ to always break the law, it is $M$ 's best reply to monitor.

Since for "high" values of private benefit $\left(\pi>\frac{1}{1-\beta}\right)$, the activity may be declared illegal, when does $A$ end up going to jail? The answer turns out to be "never". To see this, recall that in our model with corrupt and extorting $M$, an offending $A$ can be punished in two ways: either she pays a bribe to $M$, or she spends a certain amount of time in jail. The 
first punishment merely represents a transfer of money between two risk-neutral agents and is therefore welfare neutral. The second punishment, however, represents a loss in $A$ 's utility which is not compensated by any increase in $M$ 's utility. Hence, a social planner always prefers the first over the second punishment and sets $f$ and $r$ such that, in equilibrium, $A$ never goes to jail. As noted above, the implication of this observation is that in equilibrium it must be the case that $f \geq r$, which in turn implies $F S=f-r$ and $C S=\pi+f-r$.

Consider next combinations of $(\alpha, \mu)$ that could arise in equilibrium. It follows from the proof of Remark 1 that if it is optimal to declare the activity illegal (without putting $A$ into jail), then $\alpha^{*}>0$. It is also straightforward to see from (11) that if $M$ anticipates $A$ to always abide by the law (i.e. $\alpha=1$ ), then it is optimal for him to always extort (i.e. $\mu=0$ ), which in turn makes it optimal for $A$ to break the law. We therefore have

Remark 2 There does not exist an equilibrium in which $\alpha^{*}=1$.

It follows from (10) that in any candidate equilibrium with $\alpha^{*} \in(0,1)$, we have $\mu^{*}[-$ $f+\beta \cdot(\pi+f-r)]+\left(1-\mu^{*}\right) \pi=0$. Given $\pi>0$, it implies that either $f=\beta \cdot(\pi+f-r)$ and $\mu^{*}=1$, or $f>\beta \cdot(\pi+f-r)$ and $\mu^{*} \in(0,1)$. Consequently, we have the following:

Remark 3 Implementation of equilibrium with $\alpha^{*} \in(0,1)$ requires $\mu^{*}>0$.

Remark 3 is intuitive: to induce $A$ into a (possibly) small amount of law abiding behaviour, $A$ must face a positive probability of being caught when breaking the law. If $M$ monitors, he gets $(1-\alpha)[r+(1-\beta)(\pi+f-r)]-1$, while if he extorts, he gets $r+(1-\beta)(f-r)$. Hence, $\mu^{*}>0$ if $\alpha \leq \frac{(1-\beta) \pi-1}{\beta r+(1-\beta)(\pi+f)} \equiv \tilde{\alpha}$. It cannot be optimal for $P$ to set $f$ and $r$ such that $M$ strictly prefers to monitor. For, it would mean that $A$ breaks the law too often, i.e. in that case $\alpha<\tilde{\alpha}$. $P$ could then gain by choosing a different penalty-reward structure which uniquely implements an $\alpha$ close to $\tilde{\alpha}$ and a $\mu$ close to 1. ${ }^{22}$ Hence, in equilibrium both $A$ and $M$ are indifferent between their respective two

\footnotetext{
${ }^{22}$ See Appendix for technical details.
} 
actions. Considering (10) and (11), $A$ is indifferent and $M$ is indifferent if, respectively,

$$
\mu=\frac{\pi}{\beta r+(1-\beta)(\pi+f)} \quad \text { and } \quad \alpha=\frac{(1-\beta) \pi-1}{\beta r+(1-\beta)(\pi+f)} .
$$

We now consider the impact $P$ 's choice of $f$ and $r$ might have on $A$ 's choice of $\alpha$ and $M$ 's choice of $\mu$, and then relate changes in $f$ and $r$ to changes in welfare. Observe that both $\alpha$ and $\mu$ are decreasing in $f$ and $r$. This is intuitive: the higher $f$ (or $r$ ), the higher $M$ 's incentives to extort. To make $M$ indifferent, $A$ must increase her probability of breaking the law. Similarly, the higher $f$ (or $r$ ), the lower $A$ 's incentives to break the law. To make $A$ indifferent, $M$ must decrease his probability of monitoring. Hence, if $P$ decreases $f$ (or $r$ ), this influences welfare in two ways. On the one hand, it increases the probability that $A$ will abide by the law and thus that, ceteris paribus, welfare will be equal to $\bar{w}$. On the other hand, it increases the probability that welfare will decrease by one (i.e. by the disutility of monitoring). If $\pi$ is close to $\frac{1}{1-\beta}$, the second effect dominates the first one, and, thus, any increase in $f$ (or $r$ ) increases welfare. This is easy to see: in that case and independently of $f$ and $r, \alpha^{*}$ is close to zero. Hence, any decrease in $f$ (or $r$ ) has almost no positive effect on $\alpha^{*}$. The second effect also dominates the first one if $\Delta w \equiv \bar{w}-\underline{w}$ is not too high, namely if $\Delta w \in\left(\pi, \frac{(1-\beta) \pi^{2}}{(1-\beta) \pi-1}\right)$. This is also intuitive: if $\Delta w$ is not too high it becomes relatively less important to implement law abiding behaviour by $A$. As $f$ and $r$ must be finite numbers, both $\alpha^{*}$ and $\mu^{*}$ will never be equal to zero and welfare will always be strictly less than $\underline{w}+\pi$. Hence, in this case $P$ is (strictly) better off by legalising the activity. Henceforth, we denote the interval $\left(\pi, \frac{(1-\beta) \pi^{2}}{(1-\beta) \pi-1}\right)$ as the laissez-faire region. If, however, $\Delta w$ is high (i.e. $\Delta w>\frac{(1-\beta) \pi^{2}}{(1-\beta) \pi-1}$ ), then the first effect dominates the second one, and thus any decrease in $f$ (or $r$ ) increases welfare. In this case, law abiding behaviour is set to increase welfare by a large amount and compensate for any disutility incurred by $M$.

The above discussion provides the intuition for the following formal results. 
Proposition 3 If $\pi>\frac{1}{1-\beta}$, there exists a unique equilibrium outcome in which

(i) A never goes to jail,

(ii) if $\Delta w \in\left(\pi, \frac{(1-\beta) \pi^{2}}{(1-\beta) \pi-1}\right), P$ legalises the activity, and

(iii) if $\Delta w>\frac{(1-\beta) \pi^{2}}{(1-\beta) \pi-1}, P$ declares the activity illegal; $M$ always monitors (i.e. $\mu^{*}=1$ ) and $\alpha^{*}=\frac{(1-\beta) \pi-1}{\pi}$.

Several observations follow from Proposition 3. The first observation relates to the funds which $A$ may or may not have at her disposal. If $\Delta w$ lies in the laissez-faire region and if $P$ declares the activity illegal (and optimally sets $f$ and $r$ arbitrarily high), then $P$ would only lose some $\epsilon$. However, it is important to realise that this loss is small because, by assumption, $A$ is not budget-constrained. To see this, suppose that $\beta=\frac{1}{2}, \pi=100$ and that $f=r=100,000$. It follows from (14) that in this numerical example $\mu^{*}$ and $\alpha^{*}$ are respectively close to $0.1 \%$ and $0.05 \%$. Welfare is then also close to $\underline{w}+\pi$. This numerical example, however, also implies that if $M$ decides to monitor when $A$ offends-which happens with probability $0.1 \times(1-0.005) \% \approx 0.1 \%$ - then $A$ must pay a bribe slightly in excess of 100,000 ! If $A$ is not that rich, $M$ will report the offence to $P$ who must then pay a high reward to $M$ and put $A$ in jail for a very long time. As $P$ dislikes paying $M$ a lot of money and putting $A$ in jail, she has an incentive to reduce $f$ and $r$ to take $A$ 's budget constraint into account. This reduction in $f$ and $r$, however, decreases welfare. Hence, part (ii) of Proposition 3 appears in a more robust fashion once we allow for a budget-constrained agent.

Our second observation relates to the effect the disutility of monitoring has on welfare. In our model we normalised that disutility to one. Hence, one should think of $\pi$ as

$$
\frac{A \text { 's enjoyment of (say) smoking cannabis }}{\text { disutility of monitoring }} \text {. }
$$

This implies that in our model $\pi$ decreases when it becomes more costly (in terms of effort) to uncover evidence of illegal behaviour. An identical reasoning also applies for $\Delta w$. It follows from part (iii) of Proposition 3 that $\alpha^{*}$ is nondecreasing in $\pi$ while the 
laissez-faire region is decreasing in $\pi$. Hence, welfare is nondecreasing in $\pi$ (or nonincreasing in the disutility of monitoring). This is intuitive: the higher $\pi$, the higher $M$ 's incentives to monitor (as opposed to his incentives to extort). This induces $A$ to abide by the law more often and, thus, increases $P$ 's incentives to declare the activity illegal. As a matter of fact, if $M$ has a lot of bargaining power and for high values of $\pi, \alpha^{*}$ may be close to one in which case welfare only decreases by $M$ 's disutility of monitoring.

The third observation revisits our assumption that if $M$ catches an offending agent, he gets a bigger bribe (as compared to the one he gets if he extorts), which is a crucial assumption for Propositions 2 and 3. In our model this is due to the fact that, with probability $p>0, M$ can physically prevent $A$ from getting $\pi$ (if $A$ broke the law when $M$ monitored). While we believe this to be realistic, one can also think of alternative reasons why this may be the case. For example, a law abiding person is likely to be less wealthy than someone who ran a clandestine casino (or who sold drugs) for a certain time. In such a case, if $M$ extorts, he does not know whether $A$ broke the law or not and bargaining will be plagued by private information problems. However, if $M$ monitors (when $A$ breaks the law) he knows that $A$ is very wealthy (and, thus, that he can extract a bigger bribe from her). We do not expect our main results to change if one were to analyse a (more complicated) model in which the agent's budget constraint depends on her activity choice.

Fourth, recall that we assume that $P$ can only punish $A$ by sending her to jail. This assumption is not crucial to our results. Suppose $P$ could fine the agent by incurring a small transaction cost associated with the bureaucracy of administering a fine (such as cost of a bank transfer, cost of writing a receipt, and such like). As $M$ does not incur that cost when he extorts, it would still be optimal for $P$ to "delegate" the punishment to $M$, and all our results should go through. 


\section{Ex ante and ex post corruption}

We now consider a set-up in which $A$ and $M$ may agree that $A$ will commit the offence so that, through monitoring, $M$ is able to get the evidence of $A$ breaking the law. This setup captures the Hong Kong example of the fake casino (see Introduction) where actors were employed by the police and the mafia to pose as players and subsequently were put in prison only to be let out a day after. This is what we defined in our Introduction as ex ante corruption. Of course, ex ante corruption only happens if $A$ is willing to participate in this collusive scheme. This restriction to satisfy A's participation constraint rules out the extortion scenario considered earlier, but still leaves room for ex post corruption.

To avoid confusion we outline the timing of events. At time $t=0, P$ chooses whether or not to legalise the activity. If the activity is declared legal, the game ends. If it is declared illegal, then the game continues and $P$ chooses $f$ and $r$. At $t=1, A$ and $M$ decide whether or not to engage in ex ante corruption. At $t=2$, if there is ex ante corruption, then $A$ breaks the law. If, alternatively, there is no ex ante corruption, then $A$ decides whether or not to break the law, while $M$ decides whether or not to monitor. At $t=3, A$ and $M$ decide whether or not to engage in ex post corruption, provided that there is no ex ante corruption, $A$ chose to break the law and $M$ monitored. At $t=4, M$ makes his report to $P$. At $t=5$, bribes (if any) are exchanged and payoffs are realised. We assume that both $M$ and $A$ 's outside options are equal to zero.

Proposition 4 If $\mathrm{M}$ needs $\mathrm{A}$ 's consent (or her active participation) to create the evidence of $\mathrm{A}$ 's offence, then in any equilibrium $\mathrm{P}$ declares the activity illegal, welfare is close to $\bar{w}$, while $r$ and $f$ are arbitrarily large.

The intuition behind this result goes as follows: the higher $f$, the higher the bribe that $M$ must give to $A$ to convince her to create evidence of the offence. To deter ex ante corruption, $P$ sets $f$ at a "very high" level. As $f$ is "very high", $A$, who must be breaking the law with a positive probability, so as to create incentives for $M$ to monitor, can gain from offering $M$ a bribe to hide adverse monitoring evidence. Hence, in equilibrium $P$ avoids 
ex ante but not ex post corruption. For the same reason as outlined in Section 3 (p. 17), $A$ randomises between breaking and not breaking the law to make $M$ indifferent between monitoring and not monitoring, which in equilibrium requires $1-\alpha^{*}=\frac{1}{\beta r+(1-\beta)(\pi+f)}$. Similarly, $M$ must also make $A$ indifferent, which requires $\mu^{*}=\frac{\pi}{\beta r+(1-\beta)(\pi+f)}$. The higher $f$ or $(r)$, the higher is the bribe that $A$ must pay to $M$ to conceal monitoring evidence of $A$ 's offence. To make $M$ indifferent, $A$ must then break the law less often. To make $A$ indifferent, $M$ must then monitor less. If $f=r \rightarrow \infty, P$ implements an equilibrium in which $A$ almost always abides by the law while $M$ almost never monitors. Hence, in any other equilibrium, welfare must also be close to first best.

To come back to our Hong Kong example, in this section we crucially assumed that all actors must at least get their outside options. In the real world, however, some actors may have been forced at gunpoint to participate in such an ex ante collusive scheme. If the mafia can use violence to intimidate actors and force them to participate in an illegal activity, then $A$ 's participation constraint becomes irrelevant and all the results of our previous section go through.

\section{Conclusion and some policy implications}

Conventional wisdom suggests that policy makers should declare activities illegal if they are very harmful from a social point of view. We show that this wisdom is incomplete if the police force can both extort and engage in ex post corruption. In particular, our model highlights that $\pi$ (the private benefit or the profitability of the undesirable activity) is a more important variable than $\Delta w$ (the harmfulness of the undesirable activity): if $\pi$ is not "high", policy makers should legalise the undesirable activity (independently of $\Delta w)$.

In this paper we only considered the "one-monitor case". Other papers (for example, Kofman and Lawarrée, 1993; Polinsky and Shavell, 2001; Khalil and Lawarrée, 2003) have analysed the two-monitor case under the assumption that the second monitor is 
"corruption-free". Our paper highlights the importance of such a second monitor: if there is only one monitor, for a large range of parameter values the social planner cannot gain by declaring the activity illegal. However, special care must be paid to ensure that the second monitor will not collude with the first one: simply assuming that the second monitor is honest rules out this interesting possibility by assumption.

We showed that a police officer has more incentives to monitor for high values of $\pi$. To see how this insight can be useful for a policy maker, consider the Hong Kong governor who wants to deter illegal gambling activities. Suppose also that the gambling mafia can force actors to participate in ex ante corruption schemes by pointing a gun to their head (i.e. all our results of Section 3 apply). There are both small and large illegal casinos. The small casinos make few profits, while the large ones make a lot of profits. There are two types of police officers: honest and dishonest. The honest ones do not create fake evidence, nor engage in ex post corruption; the dishonest ones behave like the monitor in our model. Should the governor encourage the honest police officers to detect the small or the large clandestine casinos? Our model suggests that the governor may prefer the former. This is because dishonest police officers already have a lot of incentives to go after the large casinos as they then get a large bribe. Moreover, by eliminating the small casinos, the governor increases the profitability of the large ones, which in turn induces the dishonest police officers to monitor even more. As the results of our analysis suggest, when the gain of monitoring goes up, then the agent abides by the law more often to make the monitor indifferent. ${ }^{23}$ In our example it implies that, in equilibrium, fewer people will participate in illegal gambling activities.

Another observation relates to the size of rewards and punishments in the presence of corruption and extortion. As follows from our analysis in Section 3, the gain of extorting increases faster in $f$ and $r$ than the gain of monitoring. This is intuitive: if $M$ monitors, he will only receive a bribe if $A$ broke the law. If $M$ extorts, however, he gets a bribe

\footnotetext{
${ }^{23}$ The inverse relationship between incentives to break the law vis-à-vis monitoring is very robust and should also be present in a more complicated model when players play, e.g., Bayesian Nash equilibria.
} 
with probability one. Hence, in the presence of extortion it is optimal for policy makers to give low rewards to $M$ and to impose low punishments on $A .{ }^{24}$

Finally, we have also shown that welfare cannot decrease in the bargaining power of the monitor. ${ }^{25}$ Bargaining with mafia leaders is a potentially dangerous job and it is reasonable to assume that a police officer's bargaining power increases with his deterring capacity (such as the quality of his gun, etc.). Hence, one should give good guns to bad cops.

\section{Appendix}

\section{Proof of Proposition 1}

Suppose $P$ declares the activity illegal. It is clear that $\alpha^{*}<1$ : otherwise if $\alpha^{*}=1$ then $\mu^{*}=0$ which in turn implies that $\alpha^{*}=1$ cannot be $A$ 's best response. It follows that in an equilibrium with $\alpha^{*}>0$, it must be true that $\alpha^{*} \in(0,1)$ which from (3) gives $\mu^{*}=\frac{\pi}{\pi+f}$. Since $f \geq 0$ and $\pi>0$, it implies $\mu^{*}>0$. Taking into account (4), $\mu^{*}>0$ only if $1-\alpha^{*} \geq \frac{1}{r}$. If $1-\alpha^{*}>\frac{1}{r}$, then $\mu^{*}=1$, and given that $P$ 's objective function is decreasing in $r, P$ can increase her payoff by decreasing $r$ until the point where $1 / r=1-\alpha^{*}$ without affecting economic behaviour. Therefore, if $\alpha^{*}>0$, then $1-\alpha^{*}=\frac{1}{r}$. Thirdly, substitution of $\mu^{*}=\frac{\pi}{\pi+f}$ and $\alpha^{*}=1-\frac{1}{r}$ into the objective function gives $\max _{\{f, r\}} \bar{w}-\frac{\Delta w}{r}-\frac{2 \pi}{\pi+f}$. The objective function is maximised when $f$ and $r$ are arbitrarily large. Then, welfare is close to $\bar{w}$ which is greater than $\underline{w}+\pi$, the payoff she gets by legalising the activity.

\footnotetext{
${ }^{24}$ The optimality of "low" rewards and punishments has also been noted (albeit for different reasons) by Kofman and Lawarrée (1993) and by Kugler et al. (2005).

${ }^{25}$ This was also noted by Polinsky and Shavell (2001).
} 


\section{Proof of Proposition 3}

When $\alpha^{*} \in(0,1)$ and $\mu^{*} \in(0,1]$ (as follows from Remarks 2 and 3), then there arise three mutually exclusive scenaria:

(I) $A$ never goes to jail (i.e. $f \geq r$ which implies that $C S>F S \geq 0$ )

(II) if $A$ offends and is caught then she bribes $M$, while if $A$ is framed, she goes to jail (i.e. $r>f \geq r-\pi$ which implies that $C S \geq F S=0$ )

(III) $A$ goes to jail whenever either she is framed or she offended and is caught (i.e. $r-\pi>f$ which implies that $C S=F S=0$ )

Consider Scenario (III). It follows from (11) that when $C S=F S=0, \mu^{*}=0$. This is intuitive: in this case $M$ gets $r>0$ every time he discovers proof of the offence. As monitoring is a costly and risky activity, $M$ always prefers to plant evidence of the offence. As $\mu^{*}=0$ this leads $A$ to choose $\alpha^{*}=0$. This outcome yields a payoff to $P$ equal to $\underline{w}+\pi-(r+f)$, which is lower than what she would get if the activity is legalised. Scenario (III) can therefore be discarded.

We now break the proof in two steps. First, we tackle the case in which $\alpha^{*} \in(0,1)$ and $\mu^{*} \in(0,1]$ (ie $A$ is indifferent between breaking and abiding by the law, while $M$ weakly prefers monitoring over extorting). Within this step we prove the results stated in parts (i), (ii) and (iii) of Proposition 3. Second, to show uniqueness of the equilibrium outcome, we tackle the case in which $\alpha^{*} \in(0,1)$ and $\mu^{*}=1$ (ie $A$ is indifferent between breaking and abiding by the law, while $M$ strictly prefers to monitor) and demonstrate that this case results in a strictly lower welfare than the one that $P$ can achieve in the case analysed in step 1.

Step 1: Let $W_{I}\left(\alpha^{*}, \mu^{*}\right)$ and $W_{I I}\left(\alpha^{*}, \mu^{*}\right)$ denote the level of social welfare that can be achieved under Scenario (I) and (II), respectively. We show in this step that the highest value of $W_{I I}$ can never exceed the highest value of $W_{I}$, thus establishing part (i) of Proposition 3. Additionally, we show that the comparison of the highest value of $W_{I}$ 
and the welfare achieved by legalising the activity, $\underline{w}+\pi$, depends on the value of $\Delta w$, as stated in parts (ii) and (iii) of the proposition.

Write $P$ 's problem under Scenario (I) and call it Programme I $\left(P_{I}\right)$ :

$$
\begin{gathered}
\left(P_{I}\right) \max _{f, r} \quad\left\{W_{I}\left(\alpha^{*}, \mu^{*}\right)=\underline{w}+\alpha^{*} \Delta w-\mu^{*}+\left(1-\alpha^{*}\right) \pi\right\} \\
\text { s.t. } \quad\left(1-\mu^{*} \alpha^{*}\right) r-\mu^{*}+(1-\beta)\left[\mu^{*}\left(1-\alpha^{*}\right) \pi+\left(1-\mu^{*} \alpha^{*}\right)(f-r)\right] \geq 0 \\
\mu^{*}=\frac{\pi}{\beta r+(1-\beta)(\pi+f)} \\
1-\alpha^{*}=\frac{1+(1-\beta) f+\beta r}{\beta r+(1-\beta)(\pi+f)} \\
r \leq f \\
r \geq \pi-\frac{1-\beta}{\beta} f \\
f, r \geq 0
\end{gathered}
$$

$\left(P_{I}\right)$ restates programme (8)-(13) by taking into account (19), which characterises this scenario, as well as Remarks 2 and 3, which lead to (18) and (17). (20) arises from the restriction that $\mu^{*}$, given by $(17)$, must lie in $(0,1]$, while (16) ensures $M$ 's participation. Note that (19) and (20) are compatible (can be jointly satisfied) if, additionally, $\beta \pi \leq f$. Under Scenario (II), $P$ 's problem can be rewritten as the following programme $\left(P_{I I}\right)$ :

$$
\begin{gathered}
\left(P_{I I}\right) \max _{f, r} \quad\left\{W_{I I}\left(\alpha^{*}, \mu^{*}\right)=\underline{w}+\alpha^{*} \Delta w-\mu^{*}+\left(1-\alpha^{*}\right) \pi-(f+r)\left(1-\mu^{*}\right)\right\} \\
\text { s.t. } \quad\left(1-\mu^{*} \alpha^{*}\right) r-\mu^{*}+(1-\beta) \mu^{*}\left(1-\alpha^{*}\right)[\pi+f-r] \geq 0 \\
\mu^{*}=\frac{\pi}{\beta r+(1-\beta)(\pi+f)} \\
1-\alpha^{*}=\frac{1+r}{\beta r+(1-\beta)(\pi+f)} \\
r \leq \pi+f \\
r>f \\
r<\pi+f-\frac{1}{1-\beta} \\
r \geq \pi-\frac{\beta}{1-\beta} f \\
f, r \geq 0
\end{gathered}
$$


$\left(P_{I I}\right)$ similarly restates (8)-(13) by taking into account (26) and (27) which characterise Scenario (II). Additionally, (28) and (29) ensure that $\mu^{*}$ given by (24) lies in $(0,1]$ and $\alpha^{*}$ given by $(25)$ is in $(0,1)$.

Observe that $W_{I}(\alpha, \mu) \geq W_{I I}(\alpha, \mu)$, so $P$ can only gain by solving $\left(P_{I I}\right)$ instead of $\left(P_{I}\right)$ if it is easier for her to implement a high $\alpha$, whenever $A$ is put in jail after being framed. Fix an arbitrary value for $\mu$. Consider (17) [same as (24)] and rewrite it in terms of $f$ as follows

$$
f=\frac{\pi}{1-\beta} \cdot\left(\frac{1-\mu}{\mu}+\beta\right)-\frac{\beta}{1-\beta} \cdot r
$$

(31) defines a line in a two dimensional $(f, r)$-space. For a given $\mu$, what kind of $\alpha^{*}$ 's would $P$ want to implement in $\left(P_{I}\right)$, given (31) and the inequality constraints (19)-(21)? Note that the higher $\alpha^{*}$, for any given $\mu$ in $\left(P_{I}\right)$, the higher the welfare.

Suppose $P$ sets $f$ such that $f \geq \beta \pi$. Then the inequality constraints in each of $\left(P_{I}\right)$ and $\left(P_{I I}\right)$, can be summarised as follows:

$$
\begin{array}{ll}
\left(P_{I}\right) \text { inequality constraints are: } & \pi-\frac{1-\beta}{\beta} f \leq r \leq f \\
\left(P_{I I}\right) \text { inequality constraints are: } & f<r<\pi+f-\frac{1}{1-\beta}
\end{array}
$$

Using (31) and (18), we can rewrite:

$$
W_{I}(\mu)=\underline{w}+\pi+\frac{(1-\beta) \pi-1}{\pi} \cdot \mu \cdot(\Delta w-\pi)-\mu
$$

Observe that under $f \geq \beta \pi$ and (32), $\mu$ can only take values in $(0,1]$. If $f=r=\beta \pi$, then $\mu=1$. If $f=r \rightarrow \infty$, then $\mu \rightarrow 0$. Moreover, if $\partial W_{I} / \partial \mu>0$, then $\mu^{*}=1$, otherwise if $\partial W_{I} / \partial \mu<0$, then $\mu^{*} \rightarrow 0$. It is easy to check that $\partial W_{I} / \partial \mu>0$ if and only if $\Delta w>\frac{(1-\beta) \pi^{2}}{(1-\beta) \pi-1}$. If $\Delta w \in\left(\pi, \frac{(1-\beta) \pi}{1-\beta-1 / \pi}\right)$, then $\partial W_{I} / \partial \mu>0$ and in order to increase the expected welfare $P$ will set $f$ and $r$ as high as possible while still respecting (19). Then as $r^{*}, f^{*} \rightarrow \infty$ we have $\alpha^{*} \rightarrow 0, \mu^{*} \rightarrow 0$ and the expected welfare when the activity is declared illegal is bounded above by $\underline{w}+\pi$ which equals the expected welfare when the activity is legalised. As both $f$ and $r$ must be finite this implies that welfare will always be strictly lower than $\underline{w}+\pi$ and thus $P$ strictly prefers to legalise the activity. 
Note that while $\mu^{*}$ is given by the same expression in both $\left(P_{I}\right)$ and $\left(P_{I I}\right)$, the value of $\alpha^{*}$ differs. For convenience, we use $\alpha_{I}^{*}$ and $\alpha_{I I}^{*}$ to distinguish the two. Substituting (31) into (18) and (25), and re-arranging, we have, respectively:

$$
\alpha_{I}(\mu)=\left(1-\beta-\frac{1}{\pi}\right) \mu \quad \text { and } \quad \alpha_{I I}(r, \mu)=1-\frac{\mu}{\pi}(1+r)
$$

Note that $\alpha_{I}(\mu)$ does not depend on $r$. Further, substituting $\alpha_{I I}(r, \mu)$ from (35) together with (31) into (22) and then partially differentiating, it can be checked that

$$
\partial W_{I I}(r, \mu) / \partial r=\mu \cdot\left\{(1-\mu) \frac{2 \beta-1}{1-\beta}-\frac{\Delta w-\pi}{\pi}\right\}
$$

If $\beta \leq 1 / 2$, the derivative is negative and, for $\forall \mu \in(0,1)$, one wants to set $r$ as low as possible in the feasible set. If $\beta$ is sufficiently high, the derivative is positive and one wants to increase $r$ as much as possible in the feasible set. It therefore follows that one can always obtain any $\mu$ at a corner solution.

To find the corner points in the feasible set of $\left(P_{I I}\right)$ when $f \geq \beta \pi$, plot the latter together with (33) and (31). There exist three corner points, which we define as follows:

$$
\begin{array}{ll}
\text { Point A : } & (31) \text { and } f=\frac{1}{1-\beta}-\pi+r \\
\text { Point B : } & (31) \text { and } f=r \\
\text { Point C : } & (31) \text { and } f=\beta \pi
\end{array}
$$

It can be checked that

$$
\begin{array}{ll}
\text { at point } \mathrm{A}: & r^{A}(\mu)=\frac{\pi}{\mu}-1 \\
\text { at point } \mathrm{B}: & r^{B}(\mu)=\beta \pi+\frac{1-\mu}{\mu} \cdot \pi \\
\text { at point } \mathrm{C}: & r^{C}(\mu)=\beta \pi+\frac{1-\mu}{\mu} \cdot \frac{\pi}{\beta}
\end{array}
$$

and further, that for a given $\mu \leq 1$

$$
\begin{array}{ll}
\alpha_{I I}\left(r^{A}(\mu), \mu\right)=0 & <\alpha_{I}(\mu) \\
\alpha_{I I}\left(r^{B}(\mu), \mu\right)=\left(1-\beta-\frac{1}{\pi}\right) \mu & =\alpha_{I}(\mu) \\
\alpha_{I I}\left(r^{C}(\mu), \mu\right)=1-\frac{\mu}{\pi}-\frac{1-\mu}{\beta}-\beta \mu & \leq \alpha_{I}(\mu)
\end{array}
$$


Suppose now that, when solving $\left(P_{I I}\right), P$ chooses $f$ such that $f<\beta \pi$. Then inequality constraints in $\left(P_{I I}\right)$ become:

$$
\begin{aligned}
\pi-\frac{1-\beta}{\beta}<r & <\pi+f-\frac{1}{1-\beta} \\
\frac{\beta}{1-\beta}<f & <\beta \pi
\end{aligned}
$$

It is straightforward to show that under (31) the first inequality sign in (46) is satisfied and hence can be ignored. Analysis of the remaining inequalities in (46) and (47) together with (31) suggests that the two corner points, Point A and Point C, identified earlier arise in this case also. Noting that in $(r, f)$-space the coordinate of Point $\mathrm{A}, f^{A}$, is $\frac{1-\mu}{\mu} \pi+\frac{\beta}{1-\beta}$, and that it is greater than $\frac{\beta}{1-\beta}$, we conclude that Points $\mathrm{A}$ and $\mathrm{C}$ are the only two corner points possible in this case. But, as checked earlier, either of these corner points implements $\alpha_{I I}(r, \mu) \leq \alpha_{I}(\mu)$.

Step 2: Suppose there exists an equilibrium outcome in which the following three conditions are satisfied: $f \geq \beta \pi, r=\pi-\frac{1-\beta}{\beta} f$ and $\mu^{*}=1$. From our computations in Step 1 above we know that $M$ strictly prefers to monitor if $\alpha^{*}<\frac{(1-\beta) \pi-1}{\pi}$. This implies that $P$ is achieving a payoff strictly lower than the one summarized in (34). In this case $P$ can profitably deviate by setting $r=f=\beta \pi+\epsilon$. As $\epsilon \rightarrow 0$, this yields a payoff to $P$ which is equal to the one summarized in (34). Hence, there does not exist an equilibrium outcome in which $\alpha^{*} \in(0,1)$ and in which $M$ strictly prefers to monitor.

\section{Proof of Proposition 4}

For ex ante corruption to take place, it is $M$ who will have to offer a bribe to $A$, since $A$ is certain to be fined when ex ante corruption is agreed and the adverse evidence is created and reported. If ex ante corrupt deal goes ahead and consequently the evidence of the offence is reported, then $A$ gets $-f+b r i b e$, while $M$ gets $r$-bribe, which generates the joint surplus of $r-f$. If there is no ex ante corruption, $A$ and $M$ may still choose to engage in ex post corruption (in the case when $A$ offended while $M$ monitored). Then taking into account the possibility of ex post corruption, the expected payoffs in 
the absence of ex ante corruption are $(1-\alpha)[(1-\mu) \pi+\mu(-f+\beta \cdot C S)]$ to $A$ and $\mu[(1-\alpha)(r+(1-\beta) \cdot C S)-1]$ to $M$, and these sum up to $(1-\alpha)(1-\mu) \pi+\mu(1-$ $\alpha)[r-f+C S]-\mu$. Ex ante corruption will take place if the latter is smaller than the joint ex ante corruption surplus, $r-f$. This is equivalent to the following inequality:

$$
(1-\mu(1-\alpha))(\pi+f-r)+\mu(1-\alpha) C S-\alpha \pi-\mu<0
$$

Inspection of (48) suggests that if $P$ sets $f$ and $r$ such that $\pi+f-r<0$ (and consequently $C S=0$ ), then the ex post corruption is deterred but the ex ante corruption will take place. Alternatively, if $C S=\pi+f-r>0$ then the ex ante corruption, but not the ex post corruption, can be prevented. Setting $\pi+f-r=0, P$ can prevent both ex ante and ex post corruption but only if $\alpha=\mu=0$. This, of course, cannot be optimal for $P$ : allowing ex ante corruption will result in the welfare $(=\underline{w}-r-f)$ which is necessarily less than the welfare when $A$ 's activity is legalised; allowing ex post corruption, $P$ can achieve the welfare which may exceed the welfare when the activity is legalised. Hence, $P$ will set $f$ and $r$ so as to rule out ex ante corruption, albeit at the expense of allowing ex post corruption. With $\pi+f-r>0$, the constraint to ensure deterrence of ex ante corruption, ie the opposite of (48), becomes $(1-\alpha) \pi+f-r-\mu \geq 0$.

$P$ now faces the following maximisation problem:

$$
\begin{array}{cc}
\max _{r, f} & \underline{w}+\alpha^{*} \Delta w-\mu^{*}+\left(1-\alpha^{*}\right) \pi \\
\text { s.t. } & \left(1-\alpha^{*}\right)\left[\left(1-\mu^{*}\right) \pi+\mu^{*}(-f+\beta \cdot C S)\right] \geq 0 \\
\mu^{*}\left[\left(1-\alpha^{*}\right)(r+(1-\beta) \cdot C S)-1\right] \geq 0 \\
\alpha^{*} \in \arg \max (1-\alpha)\left[\left(1-\mu^{*}\right) \pi+\mu^{*}(-f+\beta \cdot C S)\right] \\
\mu^{*} \in \arg \max \mu\left[\left(1-\alpha^{*}\right)(r+(1-\beta) \cdot C S)-1\right] \\
C S>0 \\
(1-\alpha) \pi+f-r-\mu \geq 0 \\
f, r \geq 0 \\
\alpha^{*}, \mu^{*} \in[0,1]
\end{array}
$$


This programme now takes into account participation constraints for both $A$ and $M$, given by (50) and (51).

There does not exist an equilibrium in which $\alpha^{*}=1$. For, if $\alpha^{*}=1$ then $\mu^{*}=0$ which makes it profitable for $A$ to deviate to $\alpha^{*}=0$. This insight implies that there does not exist an equilibrium in which welfare is equal to $\bar{w}$. Consider now a candidate equilibrium in which $\alpha^{*} \in(0,1)$. It follows from $(52)$ that $A$ is indifferent if

$$
\mu^{*}=\frac{\pi}{\beta r+(1-\beta)(\pi+f)}
$$

At the same time, $\mu^{*}>0$ only if from (53)

$$
1-\alpha^{*} \geq \frac{1}{\beta r+(1-\beta)(\pi+f)}
$$

Suppose $f=r \rightarrow \infty$ and that $\mu^{*}=\frac{\pi}{\beta r+(1-\beta)(\pi+f)}$ while $1-\alpha^{*}=\frac{1}{\beta r+(1-\beta)(\pi+f)}$. This punishment-reward scheme satisfies all the constraints and yields a welfare close to $\bar{w}$. Hence, in any other equilibrium of our game welfare must also be close to $\bar{w}$.

\section{References}

Carrillo J. Corruption in hierarchies, Annales d'Economie et de Statistique 2000a; 59; $37-61$.

Carrillo J. Grafts, bribes and the practice of corruption, Journal of Economics \& Management Strategy 2000b; 9(2); 257-286.

Hindriks J, Keen M, Muthoo A. Corruption, extortion and evasion, Journal of Public Economics 1999; 74; 395-430.

Kessler A. On monitoring and collusion in hierarchies, Journal of Economic Theory 2000; 91(2); 280-91.

Khalil F, Lawarrée J. Incentives for corruptible auditors in the absence of commitment, mimeo 2003. 
Klitgaard R. Controlling corruption. University of California Press: Berkeley and Los Angeles, California; 1988.

Kofman F, Lawarrée J. Collusion in hierarchical agency, Econometrica 1993; 61(3); 629 56.

Kugler M, Verdier T, Zenou Y. Organized crime, corruption and punishment, Journal of Public Economics 2005; 89; 1639-1663.

Mauro P. Corruption and growth, Quarterly Journal of Economics 1995; 110(3); 681-712.

Mishra A. Hierarchies, incentives and collusion in a model of enforcement, Journal of Economic Behavior and Organization 2002; 47; 165-178.

Polinsky A M, Shavell S. Corruption and optimal law enforcement, Journal of Public Economics 2001; 81(1); 1-24.

Strausz R. Delegation of monitoring in a principal-agent relationship, Review of Economic Studies 1997; 64(3); 337-57.

Tirole J. Hierarchies and bureaucracies: on the role of collusion in organizations, Journal of Law, Economics and Organization 1986; 2(2); 181-214. 